\section{Constant errors in the simultaneous matching of angles are not an artifact of the starting point for adjustment}

\section{A. W. MacRAE \\ University of Birmingham, Birmingham, England}

It is gratifying to have stimulated Wenderoth and Johnson (1982) to carry out four interesting experiments, although it appears that some of their comments arise from a misreading of the paper by MacRae and Loh (1981). I shall begin by clarifying those aspects of the paper that seem to have misled Wenderoth and Johnson and then report an analysis of some of our data in more detail than seemed to be warranted at the time the MacRae and Loh paper was written.

The problem we studied was the frequently reported finding that subjects who have been asked to reproduce an angle make consistent errors such that acute angles are reproduced larger than they should be and obtuse angles are made too small. We argued that no purely perceptual error could account for such results and that attention had to focus on artifacts of experimental procedure or analysis. The results we obtained were a complete surprise to us because, in spite of eliminating likely artifacts, we found that the direction of the errors did still depend strongly on the size of the angle being matched.

We had designed the study to try to pin down what we believed to be artifacts in one line of investigation. We tried to avoid some and to evaluate the contribution of others. Delay between viewing the standard and the variable looked like a prime candidate, as did relative position of the angles, orientation of the angle midlines, and the starting point for adjustment. In our experiment, we found the first three to be important, but not the fourth.

Wenderoth and Johnson believe that conclusion to have resulted from a misinterpretation of our data. They think that the surprising effect of angle size on the preferred direction for errors of adjustment was an artifact of the starting position from which our subjects began their adjustments of the variable angle. That is not so, as our paper should have made clear. Since we evidently did not make the conclusion sufficiently plain, a more elaborate treatment of the data is given below.

Wenderoth and Johnson chide us for not saying whether or not our subjects were permitted to "bracket" the final setting point-that is, to adjust

The author's mailing address is: Department of Psychology, University of Birmingham, Birmingham B15 2TT, England. the variable angle inwards and outwards before signaling that a satisfactory setting had been achieved. That point had better be dealt with first.

Our instructions made no reference to bracketing, and we did not monitor the subjects' strategies. Wenderoth and Johnson make a good case for doing so, and we shall do so in the future. From informal observation, I would guess that most subjects did bracket, and we always envisaged that they would do so-that was the purpose in giving them full control of the response angle. The apparatus strongly encouraged it, and I suspect that any subjects who did not adopt a bracketing strategy were probably not really trying to achieve a good performance. Perhaps that is one cause of the greater variability that Wenderoth and Johnson found with subjects who did not bracket.

\section{The Starting-Point (SP) Effects Reported by MacRae and Loh (1981)}

I find it more surprising that Wenderoth and Johnson think that we did not present any data on SP effects. We said, "The starting point had little impact on the simultaneous condition $(z<1$ for the main effect and for all its interactions with other factors, including all the trends relating to angle), although it exerted much more influence in the delayed reproduction conditions ..." (p. 345). Although that statement is brief, it really says a great deal about the SP effects as represented in our data. Noting that the contrasts were not just nonsignificant but were very small $(z<1$, while the $z$ for the angle effect was 9.56), it says that, on average, there was no difference between the SPs averaged over all other conditions (the main effect) and, furthermore, that there was no differential effect of SP for different angles, spatial arrangement, orientation, or any combination of these factors, but that there were effects (admittedly unspecified) when delay was introduced.

The big surprise and puzzle in our results was the error in the simultaneous condition, and the paper was almost entirely concerned with that. In the simultaneous condition, SP played virtually no part.

The artifact that they envisage is based on the supposition that there is both a main effect of angle and an interaction of SP with angle, as in their Figure 1. (Although, of course, they interpret the main effect of "angle"' as an artifact of that analysis.)

In fact, that proposal is directly contradicted by the results that we reported. Their parameterization of the data as "angle," "proximity of starting point," and the interaction between these main ef- 
fects is different from ours only in its labeling. It does not invoke any new contrasts, but merely renames those we had examined.

Let us adopt the following terminology in which a letter denotes a particular combination of stimulus angle and SP: A denotes a 20-deg angle and 10-deg SP; B denotes a 20-deg angle and 170-deg SP; C denotes a 160-deg angle and 10-deg SP; D denotes a 160-deg angle and 170-deg SP.

In terms of the proximity of SP, as invoked by Wenderoth and Johnson, $A$ and $D$ have proximal SPs and B and C have distal SPs.

In the corresponding MacRae and Loh parameterization, the components "angle," "starting point," and "angle $X$ starting point" correspond to the contrasts $(A+B)$ vs. $(C+D),(A+C)$ vs. $(B+D)$, and $(A+D)$ vs. $(B+C)$, respectively.

The components of "angle," "proximity," and "angle $X$ proximity," as discussed by Wenderoth and Johnson, correspond to $(A+B)$ vs. $(C+D)$, $(A+D)$ vs. $(B+C)$, and $(A+C)$ vs. $(B+D)$, respectively. Thus, their analysis substitutes the label "proximity" for our term "angle $x$ starting point" and the label "angle $X$ proximity" for our term "starting point." We reported both of these components to be nonsignificant and very small.

If both were substantial, but only just not significant, then some alternative parameterization could possibly prove significant, although not the one that they propose, because it does not combine the data in any new arrangement. But since we reported the angle effect to account for much more variance than the other two combined ( $\mathrm{z}$ for angle being 9.56, compared with two $\mathrm{zs}<1$, all having the same error term), it is impossible for any restructuring to eliminate it.

\section{Further Analysis of the SP Effects}

Since a mere statement of a nonsignificant difference is clearly not sufficiently convincing, Figure 1 shows a more detailed analysis of our results, concentrating on the possibility of a SP effect. The analysis was carried out using the Royal Statistical Society's GLIM program for the analysis of general linear models (Baker \& Nelder, 1978). The data consist of the proportions of angle settings that were larger than the angle being matched, and the error distribution is taken to be binomial.

In any comparison with the figures in Wenderoth and Johnson (1982), it should be noted that they adopt the convention of designating angles that the subject set too large as negative errors, whereas the convention here follows that used in MacRae and Loh (1981) in calling them positive errors.

The points denote the maximum-likelihood fits to the data of a model in which every combination of angle and SP is allowed to take an independent

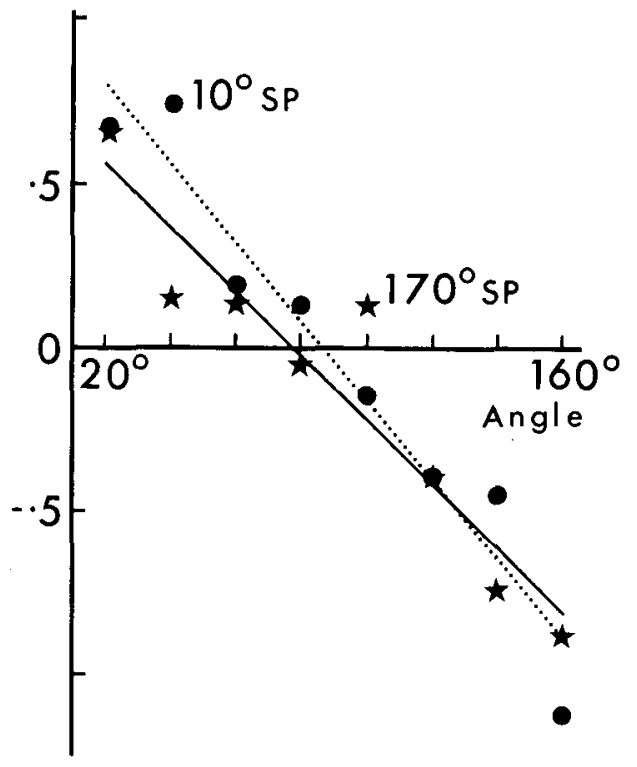

Figure 1. Maximum-likellhood fits to the data of MacRae and Loh (1981) for two models. In one, a different parameter is estimated for every combination of starting point for adjustment (SP) and angle. (Circles represent 10-deg SPs; stars represent 170-deg SPs). In the other, different straight lines are fitted as a function of angle to data obtained with a 10-deg SP (dots) and with a 170-deg SP (line). The vertical axis is expressed in logistically scaled proportions of positive errors, that is, $\log _{2}[P /(1-P)]$.

value. The lines show a maximum-likelihood fit to a model in which a different straight line is fitted to the data, depending on which SP was used. The difference between the lines is very far from being significant, and it is in the opposite direction to the one that they envisage. Some of the points differ in the direction they suggest, although only for the 40-deg angle is the difference significant $(z=$ $1.98, \mathrm{p}<.05$ ), and its direction is opposite to the one that they anticipate. That is, there is more "overshoot" with a SP of 10 deg than "undershoot" with a SP of $170 \mathrm{deg}$. In all other cases, $z$ is less than 1. But the effect of angle is almost always much greater than the effect of SP, as is shown by the fact that the direction of the most frequent error is the same for both SPs, except for the two angles closest to $90 \mathrm{deg}$.

But it does seem likely that SP should have some effect on the settings that subjects make. It had a considerable effect in the delayed-comparison conditions, and in other data, as yet unpublished, we have found greater SP effects than in the MacRae and Loh study. However, a small effect cannot serve as more than a partial explanation for a large effect, and the disparity in size between SP and angle effects has been very great in all the data I have seen. Even after all possible allowance has been made for any SP effect, there is still a much larger angle effect to be explained. 


\section{Differences From the Wenderoth and Johnson (1982) Study}

The big difference between their analysis and ours is that they make use of mean errors as their basic measure, whereas we used the proportions of positive and negative errors. They may not agree with our contention that mean errors are likely to be systematically misleading in this situation, but in the present instance it hardly matters. An analogous analysis of our data in terms of mean errors leads to just the same conclusions-again, SP effects are negligible.

It appears that they have obtained SP effects that were greater in relation to the angle effects than those we found and that were generally in the opposite direction. That result is interesting. It may of course be the case that mere chance sampling effects led to the difference, but there are also several differences between their procedure and ours, and any of these may be important.

For example, their subjects operated in darkness, whereas ours were in normal room lighting. Their response angles moved in 2-deg steps in response to microswitch controls, whereas ours changed as smoothly as a $2,048 \times 2,048$ screen resolution allowed and were under the control of a rotary knob. The layout of their angles on the screen was different from ours, both in respect of the relative positions of the vertices and their relative orientations. (The open end of their horizontal angle was towards the vertex of the vertical one, whereas ours faced away from it.) Perhaps most significantly, their angles changed at a rate that was limited to $10 \mathrm{deg} / \mathrm{sec}$. That imposed a minimum duration of $15 \mathrm{sec}$ on adjustment from a distal SP and would not, perhaps, be conducive to a bracketing strategy once the angle was approximately correct. Our subjects could ad- just the angles as rapidly as they could turn the control knob.

I note these differences not to object to their procedures but to point out that differences in outcome are not altogether surprising and may warrant further investigation. Their Experiment 4 is particularly interesting in this context. It does not surprise me in the least that a different psychophysical procedure should reduce or remove the effect of angle, because the conclusion we reported, and which was forced on us by our data and by the logic of the situation, was that the systematic error tendency practically had to be an outcome of the particular response procedure used (although that does not exclude the possibility that it might occur with other procedures too, possibly for somewhat different reasons).

However, I must unrepentantly continue to claim that our results show that constant errors can occur in matched reproduction of angles even when some likely biases are eliminated. In our study, the starting point for adjustment had no more than a minor effect on the results.

\section{REFERENCES}

BAKen, R. J., \& Nelden, J. A. The GLIM system, release 3. Generalised linear interactive modelling manual. Oxford: $\mathrm{Nu}$ merical Algorithms Group, 1978.

MacRAe, A. W., \& LoH, H. K. Constant errors occur in matched reproduction of angles even when likely biases are eliminated. Perception \& Psychophysics, 1981, 30, 341-346.

Wenderoth, P., \& Johnson, M. Constant errors do not occur in the matched reproduction of angles. Perception \& Psychophysics, 1982, 32, 388-392.

(Manuscript received September 30, 1982; accepted for publication October 29, 1982.) 